\title{
Association between thyroid function and assessment of hepatic fat and iron contents by magnetic resonance imaging
}

Till Ittermann $11^{1, *}$, Rehman Mehmood Khattak ${ }^{1,2,{ }^{*},}$ Marcello R P Markus ${ }^{3}$, Jens-Peter Kühn ${ }^{4}$, Marie-Luise Kromrey5, Giovanni Targher ${ }^{6}$, Antje Steveling ${ }^{7}$, Matthias Nauck ${ }^{8,9}$ and Henry Völzke ${ }^{1}$

${ }^{1}$ Institute for Community Medicine, University Medicine Greifswald, Greifswald, Germany

${ }^{2}$ Department of Zoology, Islamia College Peshawar (CU), Peshawar, Pakistan

${ }^{3}$ Department of Internal Medicine B, University Medicine Greifswald, Greifswald, Germany

${ }^{4}$ Institute of Radiology, University Hospital, Carl-Gustav-Carus University, Dresden, Germany

${ }^{5}$ Department of Radiology and Neuroradiology, University Medicine Greifswald, Greifswald, Germany

${ }^{6}$ Division of Endocrinology, Diabetes and Metabolism, Department of Medicine, University of Verona, Verona, Italy

7Department of Internal Medicine A, University Medicine Greifswald, Greifswald, Germany

${ }^{8}$ Institute for Clinical Chemistry and Laboratory Medicine, University Medicine Greifswald, Greifswald, Germany

${ }^{9}$ DZHK (German Centre for Cardiovascular Research), Partner Site Greifswald, University Medicine, Greifswald, Germany

Correspondence should be addressed to T Ittermann: till.ittermann@uni-greifswald.de

*(T Ittermann and R M Khattak contributed equally to this work)

\section{Abstract}

The associations of thyroid function parameters with non-alcoholic fatty liver disease (NAFLD) and hepatic iron overload are not entirely clear. We have cross-sectionally investigated these associations among 2734 participants of two population-based crosssectional studies of the Study of Health in Pomerania. Serum levels of thyroid-stimulating hormone (TSH), free tri-iodothyronine (fT3), and free thyroxine (fT4) levels were measured. Liver fat content (by proton-density fat fraction) as well as hepatic iron content (by transverse relaxation rate; R2*) were assessed by quantitative MRI. Thyroid function

\section{Key Words}

- thyroid

- TSH

- $\mathrm{fT} 3$

- fT4

- hepatic steatosis

- NAFLD parameters were associated with hepatic fat and iron contents by median and logistic regression models adjusted for confounding. There were no associations between serum TSH levels and liver fat content, NAFLD, or hepatic iron overload. Serum fT4 levels were inversely associated with liver fat content, NAFLD, hepatic iron contents, and hepatic iron overload. Serum fT3 levels as well as the fT3 to fT4 ratio were positively associated with hepatic fat, NAFLD, hepatic iron contents, but not with hepatic iron overload. Associations between fT3 levels and liver fat content were strongest in obese individuals, in which we also observed an inverse association between TSH levels and NAFLD. These findings might be the result of a higher conversion of fT4 to the biologically active form fT3. Our results suggest that a subclinical hyperthyroid state may be associated with NAFLD, particularly in obese individuals. Furthermore, thyroid hormone levels seem to be more strongly associated with increased liver fat content compared to hepatic iron content.

\section{Introduction}

Non-alcoholic fatty liver disease (NAFLD) is a common finding in individuals with obesity or type 2 diabetes mellitus and its prevalence is steeply increasing worldwide (1).
Recent meta-analyses of observational studies revealed inconsistent findings regarding the association between subclinical or overt primary hypothyroidism and https://ec.bioscientifica.com

https://doi.org/10.1530/EC-21-0566

C 2022 The authors Published by Bioscientifica Ltd

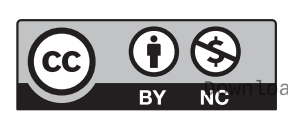

This work is licensed under a Creative Commons Attribution-NonCommercial 4.0 International License. ded from Bioscientifica.com at 04/26/2023 10:36:52AM 
NAFLD $(2,3)$. While a significant positive association was reported between variably defined hypothyroidism and NAFLD in one meta-analysis (3), no association was observed in another meta-analysis (2). Furthermore, some population-based studies showed an inverse association between serum free thyroxine (fT4) levels and NAFLD $(4,5)$. However, in only one of these studies, a positive association of thyroid-stimulating hormone (TSH) levels with NAFLD was also reported (4). Conversely, a case-control study, matching individuals with subclinical hypothyroidism to euthyroid controls, showed a positive association between serum TSH levels and NAFLD but failed to detect an association between serum fT4 levels and NAFLD (6).

A recent comprehensive review on this topic concluded that an association between primary hypothyroidism and risk of development and progression of NAFLD is likely, but that further mechanistic studies are needed to better elucidate the potential pathophysiological mechanisms underpinning this association (7). In the aforementioned studies, NAFLD was assessed either by liver ultrasound or blood biomarkers/scores $(2,3,4,5,6)$. However, a major limitation of the ultrasound examination is its inability to detect small amounts of fat infiltration and to distinguish liver fat from iron (8). MRI techniques allow an accurate quantification of liver fat and iron contents via protondensity fat fraction (PDFF) and transverse relaxation rate $\left(\mathrm{R} 2{ }^{\star}\right)$, respectively $(8,9,10)$.

As far as we know, there are no population-based studies that investigated the associations of thyroid function parameters with NAFLD and hepatic iron overload together. An association between thyroid function and hepatic iron overload may be plausible because previous studies showed increased serum ferritin levels in individuals with hyperthyroidism $(11,12)$, even though this finding was not confirmed in a populationbased study (13). Against this background, the aim of our cross-sectional study was to investigate the associations of laboratory markers of thyroid function with NAFLD and hepatic iron overload, directly assessed by MRI, in the Study of Health in Pomerania (SHIP).

\section{Materials and methods}

\section{Study population}

The SHIP project includes several large populationbased studies, which were all conducted in the Northeast of Germany. In the first SHIP cohort SHIP-START, 6265 individuals aged 20-79 years were selected from population registries, of which 4308 individuals (response 68.8\%) participated between 1997 and 2001 (14). For the present analysis, we used data from the second follow-up (SHIP-START-2), in which 2333 individuals aged 30-93 years were examined between 2008 and 2012. In parallel to SHIP-START-2, baseline examinations of a second cohort were conducted (SHIP-TREND). For SHIP-TREND, a separate stratified random sample of 8826 adults aged 20-79 years was drawn and 4420 subjects participated between 2008 and 2012 (response 50.1\%). Assessment methods were the same in SHIP-START-2 and SHIP-TREND-0 and these study waves were the first, in which MRI examinations were part of the study protocol. In both studies, all participants gave written informed consent. The study was approved by the Local Ethics Committee of the University of Greifswald and followed the Declaration of Helsinki.

Of the 6753 individuals examined in the SHIP-START-2 and SHIP-TREND cohorts, we excluded 70 individuals who had cirrhosis or chronic liver diseases and 140 individuals who admitted to drink more than $60 \mathrm{~g}$ of alcohol per day over the last month prior to examination. Furthermore, we excluded 3787 individuals who did not attend MRI examinations and 156 individuals with missing data in exposures or confounders. As a consequence of these exclusions, the total study population comprised 2600 middle-aged individuals (Fig. 1).

\section{Assessments}

Socio-demographic characteristics and history of diagnosed thyroid disorders were assessed by computerassisted personal interviews. Subjects who participated in physical training for less than $1 \mathrm{~h}$ a week were classified as being physically inactive. Mean daily beverage-specific alcohol (beer, wine, and distilled spirits) consumption was determined from alcohol intake on the last 30 days preceding the examination. Food intake patterns were selected from a validated food frequency questionnaire summarized to a dietary pattern score for each subject (15). Medication data were obtained online using the IDOM program (online drug-database leaded medication assessment) and categorized according to the Anatomical Therapeutical Chemical (ATC) classification index. Thyroid medication was defined by the ATC code H03.

Blood samples were taken non-fasting in SHIP-START-2. In SHIP-Trend, $75 \%$ of the blood samples were taken fasting. All samples were collected between 0:07 and 0:14 h and analyzed in the Institute of Clinical Chemistry and Laboratory Medicine of the University Medicine Greifswald. Alanine aminotransferase, aspartate aminotransferase,

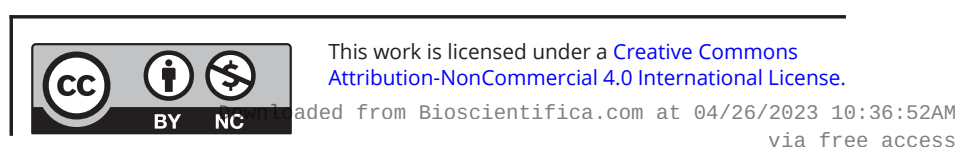




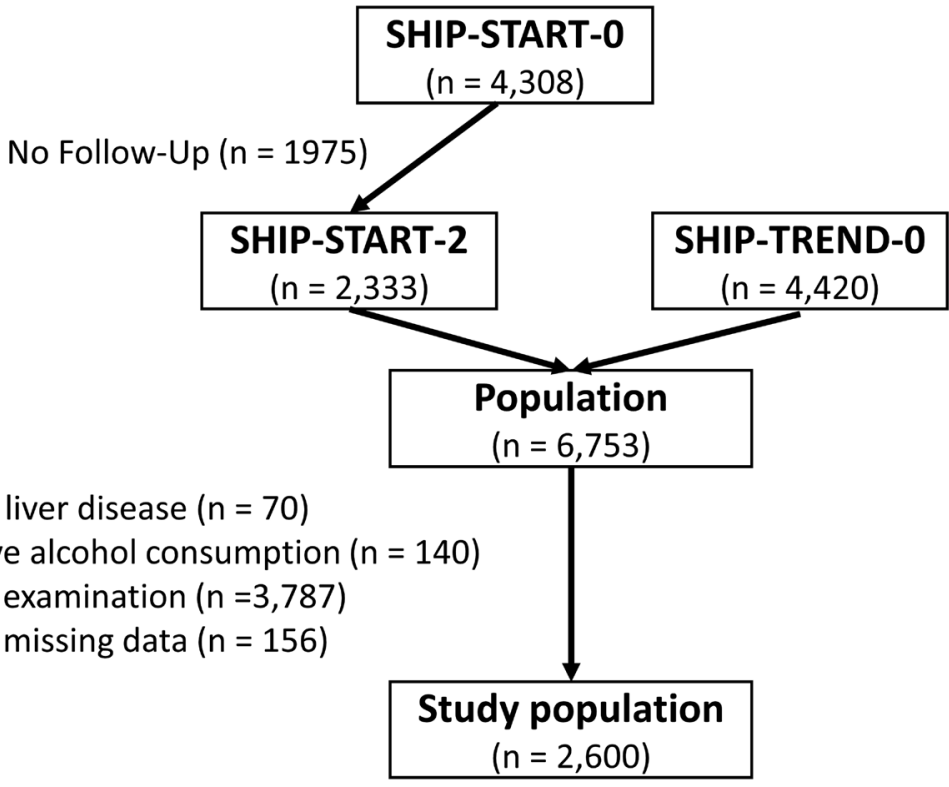

Figure 1

Consort diagram.

and $\gamma$-glutamyltransferase were measured in $\mu \mathrm{katal} / \mathrm{L}$ using the Dimension Vista 1500 analytical system (Siemens Healthcare GmbH). Serum levels of TSH, fT3, and fT4 levels were analyzed on the same platform by a homogeneous, sequential, chemiluminescent immunoassays based on LOCI $^{\circledR}$ technology (Dimension Vista ${ }^{\circledR}$, Siemens Healthcare $\mathrm{GmbH})$. The analytical measuring range for TSH was 0.005-100 $\mathrm{mIU} / \mathrm{mL}$, and the functional sensitivity was $0.005 \mathrm{mIU} / \mathrm{L}$. High and low TSH were defined according to the reference limits $0.49 \mathrm{mIU} / \mathrm{L}$ and $3.29 \mathrm{mIU} / \mathrm{L}$, which were established in SHIP-TREND (16). Likewise, high and low fT3 or fT4 were defined according to the reference ranges 3.63-6.04 pmol/L for fT3 and 10.10-16.50 pmol/L for fT4, respectively. We also calculated the ratio between fT3 and fT4 levels.

Liver MRI was performed without intravenous contrast using a 1.5-Tesla MRI system (Magnetom Avanto, VB15; Siemens Healthineers) with a 12-channel-phased-array surface coil (8). Three-dimensional chemical shift encoded gradient-echo data with three echoes and flyback readout gradient were acquired from an axial slab during a single 19-s breath-hold. Offline reconstructions of a PDFF map (including correction for $\mathrm{T} 1$ bias and $\mathrm{T} 2{ }^{*}$ decay) and a $\mathrm{R} 2 *$ map (based on $\mathrm{T} 2$ * decay measurement of PDFF) were performed (9). Parametric maps of PDFF and R2* were used for further analyses.

Mean PDFF and $\mathrm{R} 2$ * values were determined at operator-defined regions of interest placed at the center of the liver, by using Osirix (v3.8.1; Pixmec Sarl, Bernex, Switzerland) (8). NAFLD was defined by an MRI-PDFF threshold $\geq 5.1 \%$, while hepatic iron overload was defined by a threshold $\mathrm{R} 2 * 43.9$ (9).

\section{Statistical analysis}

Characteristics of the study population were reported stratified by thyroid function either as absolute numbers and percentages for categorical data or as medians, 25th, and 75th percentiles for continuous data. Thyroid function parameters (TSH, fT3, and fT4) were associated with MRIassessed hepatic fat and iron contents by median regression models adjusted for age, sex, daily alcohol consumption, BMI, physical activity, food frequency score, and time of blood sampling. We choose median regression because some of the considered outcomes were not normally distributed. Associations between thyroid biomarker concentrations and increased hepatic fat (NAFLD) and iron contents (hepatic iron overload) were analyzed by logistic regression models adjusted for the same set of confounders. Associations between thyroid biomarker concentrations and the combined endpoint of NAFLD and hepatic iron overload were analyzed by multinomial regression models with 'no NAFLD and/or hepatic iron overload' as the reference category adjusted for the same aforementioned confounders. To minimize the possible impact of outliers on the continuous exposure variables, serum TSH, fT3, and fT4 concentrations were transformed by the Royston-Sauerbrei power transformation (17), which is a linear transformation of the variables into a range between 0.2 and 1 .

All regression models were weighted for non-response to liver MRI examination. For this, inverse probability weights were calculated based on a logistic regression model with participation at the MRI examination as outcome and health-related as well as socioeconomic variables from the basic examinations as explanatory

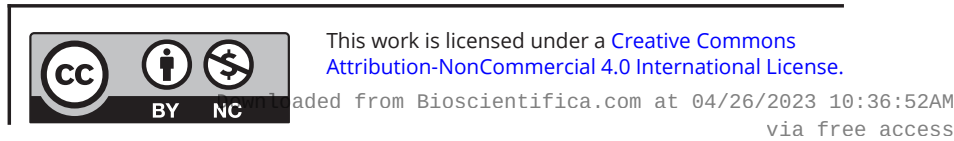


variables. Due to this procedure, the influence of individuals from groups who are more likely to drop out is weighted up, while the influence of individuals from groups who are less likely to drop out is weighted down. A $P<0.05$ was considered statistically significant. All statistical analyses were conducted with STATA 16.1 (Stata Corporation, TX, USA).

\section{Results}

In our study population, there were 239 individuals with low TSH (9.2\%) and 71 individuals with high TSH (2.7\%). Of the 239 individuals with low TSH, 53 had high fT3 or fT4 (22.1\%). Of the 71 individuals with high TSH, 6 had low fT3 or fT4 (8.5\%). Individuals with low TSH were more likely to be older and had slightly higher values for BMI and liver fat content compared to individuals with serum TSH levels in the reference range (Table 1). Individuals with high TSH were more often female and had lower median values for BMI and liver fat than individuals with serum TSH levels in the reference range. The correlation coefficient between liver fat and liver iron content was low $(\mathrm{r}=0.09)$, but liver iron content was higher in individuals with high liver fat content compared to those with low liver fat content.

After adjustment for confounding factors, we observed no significant associations of serum TSH levels with liver fat content or NAFLD, defined as MRI-PDFF liver fat content $\geq 5.1 \%$ (Table 2). Serum fT4 levels were inversely associated with liver fat content and NAFLD, while serum fT3 levels

Table 1 Characteristics of the study population stratified by serum TSH levels. Data are expressed as medians, 25th, and 75th percentile (continuous data) or as absolute numbers and percentages (categorical data).

\begin{tabular}{|c|c|}
\hline Characteristics & Normal TSH $(n=2290)$ \\
\hline Age (years) & $52(42,60)$ \\
\hline Men, n (\%) & $1104(48.2 \%)$ \\
\hline $\mathrm{BMI}\left(\mathrm{kg} / \mathrm{m}^{2}\right)$ & $27.2(24.4,30.4)$ \\
\hline Waist circumference (cm) & $89(80,99)$ \\
\hline Alcohol consumption (g/day) & $4.46(1.31,11.40)$ \\
\hline \multicolumn{2}{|l|}{ Smoking status, $\mathrm{n}(\%)$} \\
\hline Non-smoker & $916(40.0 \%)$ \\
\hline Former smoker & $843(36.8 \%)$ \\
\hline Smoker & $531(23.2 \%)$ \\
\hline Food frequency score & $14(12,16)$ \\
\hline Physically active, $n$ (\%) & $1623(70.9 \%)$ \\
\hline Glucose $(\mathrm{mmol} / \mathrm{L})$ & $5.4(5.0,5.9)$ \\
\hline $\mathrm{HbA} 1 \mathrm{c}(\%)$ & $5.3(4.9,5.6)$ \\
\hline Type 2 diabetes mellitus, $n(\%)$ & $157(6.9 \%)$ \\
\hline HDL cholesterol (mmol/L) & $1.42(1.17,1.71)$ \\
\hline LDL cholesterol (mmol/L) & $3.32(2.70,3.93)$ \\
\hline Triglycerides (mmol/L) & $1.36(0.95,1.99)$ \\
\hline Triglycerides/HDL cholesterol ratio & $0.93(0.58,1.58)$ \\
\hline Systolic blood pressure (mmHg) & $127(115,139)$ \\
\hline Diastolic blood pressure ( $\mathrm{mmHg}$ ) & $70(63,77)$ \\
\hline Hypertension, n (\%) & $1022(44.7 \%)$ \\
\hline Heart rate (bpm) & $70(63,77)$ \\
\hline High-sensitive CRP (mg/L) & $1.14(0.62,2.38)$ \\
\hline Leptin (ng/mL) & $10.2(5.7,19.8)$ \\
\hline Thyroid-stimulating hormone (mIU/L) & $1.19(0.87,1.60)$ \\
\hline Free triiodothyronine (fT3) (pmol/L) & $4.63(4.29,5.05)$ \\
\hline Free thyroxine (fT4) (pmol/L) & $13.3(12.3,14.4)$ \\
\hline fT3/fT4 ratio & $0.35(0.32,0.39)$ \\
\hline Thyroid medication use, n (\%) & $208(9.1 \%)$ \\
\hline Liver fat content, $\%$ & $3.96(2.48,8.33)$ \\
\hline NAFLDa ${ }^{\mathrm{a}}, \mathrm{n}(\%)$ & $913(39.9 \%)$ \\
\hline Liver iron content & $34.3(31.5,38.3)$ \\
\hline Hepatic iron overload ${ }^{\mathrm{b}}$ & $290(12.7 \%)$ \\
\hline Alanine aminotransferase ( $\mu \mathrm{katal} / \mathrm{L})$ & $0.39(0.29,0.53$ \\
\hline Aspartate aminotransferase ( $\mu \mathrm{katal} / \mathrm{L})$ & $0.29(0.23,0.36)$ \\
\hline$\gamma$-glutamyltranspeptidase ( $\mu$ katal/L) & $0.49(0.39,0.71)$ \\
\hline
\end{tabular}

\begin{tabular}{c}
\hline Low TSH $(n=239)$ \\
\hline $58(47,67)$ \\
$112(46.9 \%)$ \\
$27.7(24.6,31.0)$ \\
$92(81,102)$ \\
$2.61(0.65,7.57)$ \\
\\
$80(33.5 \%)$ \\
$105(44.0 \%)$ \\
$54(22.6 \%)$ \\
$14(12,17)$ \\
$158(66.1 \%)$ \\
$5.4(5.1,6.0)$ \\
$5.4(5.0,5.7)$ \\
$29(12.1 \%)$ \\
$1.42(1.17,1.68)$ \\
$3.36(2.69,4.01)$ \\
$1.29(0.92,1.81)$ \\
$0.86(0.61,1.42)$ \\
$129(114,140)$ \\
$76(71,84)$ \\
$123(51.7 \%)$ \\
$70(61,76)$ \\
$1.29(0.65,2.96)$ \\
$12.3(5.4,23.5)$ \\
$0.35(0.25,0.44)$ \\
$4.76(4.35,5.12)$ \\
$14.3(13.0,15.5)$ \\
$0.34(0.29,0.38)$ \\
$57(23.9 \%)$ \\
$4.21(2.54,9.07)$ \\
$101(42.3 \%)$ \\
$34.4(31.8,39.6)$ \\
$33(13.8 \%)$ \\
$0.37(0.29,0.52)$ \\
$0.29(0.23,0.36)$ \\
$0.50(0.38,0.68)$ \\
\end{tabular}

\begin{tabular}{c}
\hline High TSH $(n=71)$ \\
\hline $51(40,63)$ \\
$25(35.2 \%)$ \\
$26.5(22.7,29.1)$ \\
$85(76,92)$ \\
$3.13(0.73,10.23)$ \\
\\
$26(36.6 \%)$ \\
$28(39.4 \%)$ \\
$17(23.9 \%)$ \\
$15(13,18)$ \\
$54(76.1 \%)$ \\
$5.2(4.9,5.6)$ \\
$5.1(4.8,5.6)$ \\
$5(7.0 \%)$ \\
$1.41(1.25,1.70)$ \\
$3.27(2.68,3.88)$ \\
$1.50(1.11,2.08)$ \\
$1.10(0.73,1.58)$ \\
$118(110,133)$ \\
$77(69,82)$ \\
$25(35.2 \%)$ \\
$69(63,77)$ \\
$1.34(0.63,2.66)$ \\
$11.5(7.3,21.0)$ \\
$3.88(3.59,5.27)$ \\
$4.45(4.10,4.80)$ \\
$12.6(11.2,13.9)$ \\
$0.37(0.31,0.40)$ \\
$18(25.4 \%)$ \\
$3.35(2.14,6.63)$ \\
$24(33.8 \%)$ \\
$35.0(31.6,37.9)$ \\
$8(11.3 \%)$ \\
$0.37(0.25,0.53)$ \\
$0.30(0.22,0.36)$ \\
$0.46(0.35,0.67)$ \\
\end{tabular}

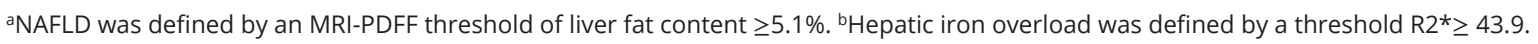

https://ec.bioscientifica.com https://doi.org/10.1530/EC-21-0566 (c) 2022 The authors Published by Bioscientifica Ltd

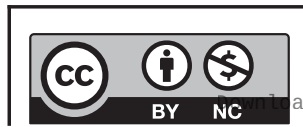

This work is licensed under a Creative Commons Attribution-NonCommercial 4.0 International License. ded from Bioscientifica com at 04/26/2023 10:36:52AM via free access 
Table 2 Associations of thyroid function parameters with liver fat content and non-alcoholic fatty liver disease (NAFLD). Data are expressed as $\beta$ coefficients and 95\% Cl from median regression models for the continuous outcome liver fat or as odds ratio and 95\%-Cl from logistic regression models for the dichotomous outcome NAFLD. Continuous exposures were power-transformed to minimize the effect of outliers on the results. All models were adjusted for age, sex, alcohol consumption, BMI, physical activity, food frequency score, and time of blood sampling.

\begin{tabular}{l}
\hline Exposure \\
\hline Power-transformed TSH \\
High TSH \\
Low TSH \\
Power-transformed fT4 \\
Power-transformed fT3 \\
Power-transformed fT3/fT4 ratio
\end{tabular}

\begin{tabular}{crr}
\hline \multicolumn{2}{c}{ Liver fat content } \\
\hline$\beta(95 \% \mathrm{Cl})$ & & $P$ value \\
\hline $0.32(-0.46,1.10)$ & & 0.426 \\
$0.26(-0.39,0.91)$ & & 0.437 \\
$-0.24(-0.60,0.12)$ & & 0.191 \\
$-1.41(-2.41,-0.42)$ & & 0.005 \\
$2.88(2.08,3.67)$ & & $<0.001$ \\
$3.50(2.27,4.73)$ & $<0.001$ \\
\hline
\end{tabular}

\begin{tabular}{ccc}
\hline \multicolumn{2}{c}{ NAFLD $^{\mathbf{a}}$} \\
\hline Odds ratio $(95 \% \mathrm{Cl})$ & & $P$ value \\
\cline { 1 - 1 } $1.16(0.58,2.32)$ & & 0.672 \\
$1.13(0.64,2.00)$ & & 0.669 \\
$0.83(0.58,1.20)$ & & 0.325 \\
$0.33(0.16,0.71)$ & & 0.004 \\
$10.08(4.59,22.15)$ & & $<0.001$ \\
$14.68(6.68,31.99)$ & $<0.001$ \\
\hline
\end{tabular}

aNAFLD was defined by an MRI-PDFF threshold of liver fat content $\geq 5.1 \%$.

were positively associated with these two outcomes (Fig. 2). The fT3/fT4 ratio was also positively associated with liver fat content and NAFLD.

There were no significant associations between serum TSH levels and hepatic iron content or hepatic iron
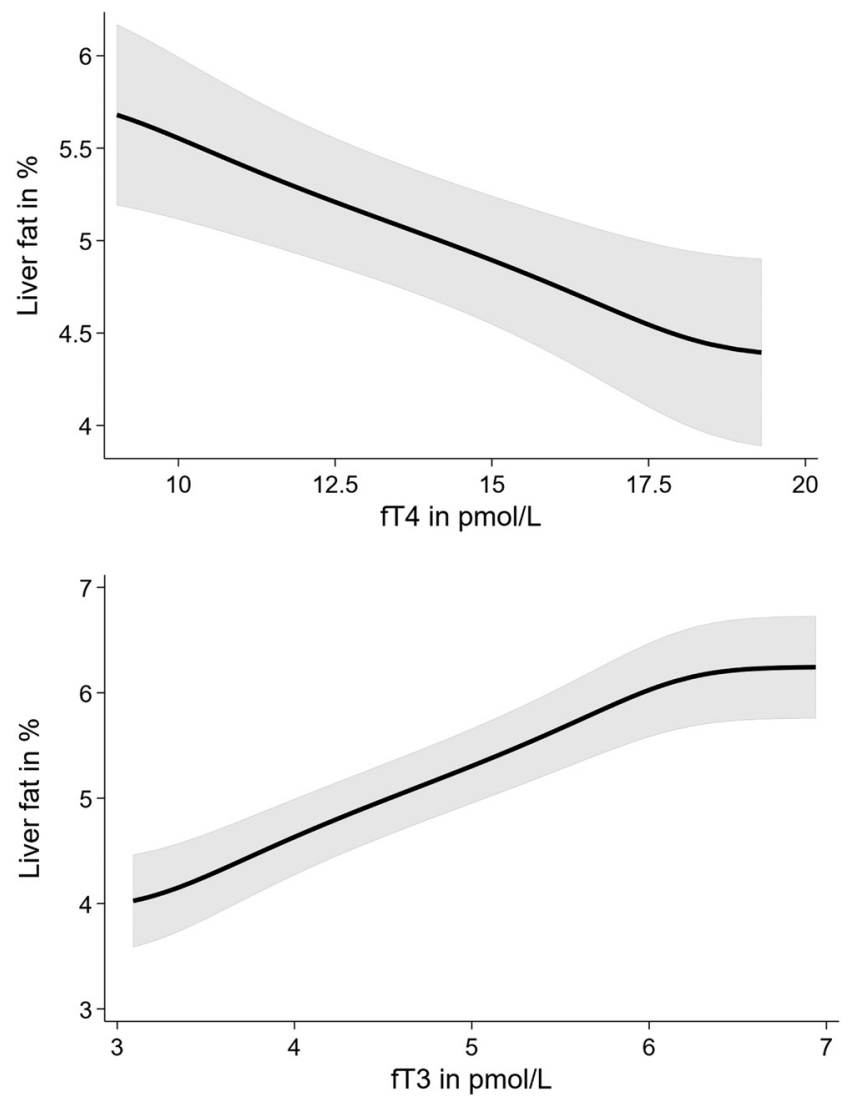

Figure 2

Associations of serum fT4 and fT3 levels with hepatic liver fat content after adjustment for age, sex, alcohol consumption, BMI, physical activity, food frequency score, and time between blood sampling and MRI examinations. Results are presented as adjusted regression curves and 95\% Cls. overload, defined as MRI-assessed R2* $\geq 43.9$ (Table 3), but we detected significant associations of serum fT3 and fT4 levels with liver iron content. Similar to the results for liver fat, serum fT4 levels were inversely associated with liver iron content, while serum fT3 levels were positively associated with this outcome. Serum fT4 levels were inversely associated with liver iron overload, but serum fT3 levels were not significantly associated with this outcome. The fT3/fT4 ratio was positively associated with liver iron content, but not with liver iron overload.

We did not find any significant associations between serum TSH levels and the combined endpoint of liver fat and iron contents (Table 4). Compared to the base category 'no NAFLD and no liver iron overload,' serum fT4 levels were inversely associated with all categories of the combined endpoint of NAFLD and liver iron overload. Serum fT3 levels were positively associated with all categories of NAFLD but not with the category where only the liver iron content was increased.

All the above-mentioned results did not differ significantly even after excluding 283 individuals taking thyroid medication. We further investigated the interactions of thyroid hormone levels with type 2 diabetes mellitus and BMI on both liver fat content and liver iron content. While there were no significant interactions of serum TSH, fT3, or fT4 levels with type 2 diabetes mellitus on either liver fat content $(P=0.371,0.397$, and 0.824 , respectively) or liver iron content $(P=0.174,0.971$, and 0.704 , respectively) in the fully adjusted regression models, the associations of serum TSH and fT3 levels with liver fat content were modified by BMI (both $P<$ 0.001). Notably, we found that liver fat content was higher in obese individuals with low TSH and high fT3, while in individuals with normal weight or overweight, the strength of the associations was attenuated (Fig. 3).

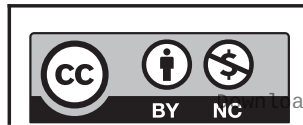

This work is licensed under a Creative Commons Attribution-NonCommercial 4.0 International License. ded from Bioscientifica.com at 04/26/2023 10:36:52AM 
Table 3 Associations of thyroid function parameters with liver iron content and liver iron overload. Data are expressed as $\beta$ coefficients and $95 \% \mathrm{Cl}$ from median regression models for the continuous outcome liver iron content or as odds ratio and $95 \% \mathrm{Cl}$ from logistic regression models for the dichotomous outcome iron overload. Continuous exposures were power-transformed to minimize the effect of outliers on the results. All models were adjusted for age, sex, alcohol consumption, BMI, physical activity, food frequency score, and time of blood sampling.

\begin{tabular}{|c|c|c|}
\hline \multirow[b]{2}{*}{ Exposure } & \multicolumn{2}{|c|}{ Liver iron content } \\
\hline & $\beta(95 \% \mathrm{Cl})$ & $P$-value \\
\hline Power-transformed TSH & $-0.92(-2.39,0.54)$ & 0.218 \\
\hline High TSH & $0.35(-0.57,1.27)$ & 0.455 \\
\hline Low TSH & $0.12(-0.84,1.08)$ & 0.808 \\
\hline Power-transformed fT4 & $-2.27(-4.00,-0.52)$ & 0.011 \\
\hline Power-transformed fT3 & $2.14(0.38,3.89)$ & 0.017 \\
\hline Power-transformed fT3/fT4 ratio & $3.22(1.58,4.87)$ & $<0.001$ \\
\hline
\end{tabular}

\begin{tabular}{ccc}
\hline \multicolumn{2}{c}{ Liver iron overload $^{\mathbf{a}}$} \\
\hline Odds ratio $(95 \% \mathrm{Cl})$ & $\frac{P \text {-value }}{1.18(0.47,2.94)}$ & 0.723 \\
$1.02(0.40,2.57)$ & & 0.969 \\
$1.04(0.68,1.61)$ & & 0.850 \\
$0.35(0.13,0.93)$ & & 0.035 \\
$0.74(0.24,2.29)$ & 0.599 \\
$2.16(0.79,5.93)$ & 0.134 \\
\hline
\end{tabular}

aLiver iron overload was defined by a threshold $R^{2 \star} \geq 43.9$.

In non-obese individuals, there was a positive association between serum TSH levels and liver fat content. Finally, we observed no significant interaction of fT4 levels with BMI on liver fat content $(P=0.108)$ as well as no significant interactions of TSH, fT3, and fT4 with BMI on liver iron content $(P=0.992,0.374$, and 0.941 , respectively).

\section{Discussion}

In our population-based study of German middle-aged individuals, we observed no significant associations of serum TSH levels with NAFLD or hepatic iron overload as determined by MRI. Serum fT3 levels were positively associated with NAFLD but not with hepatic iron overload, whereas serum fT4 levels were inversely associated with both NAFLD and hepatic iron overload as determined by MRI. Interaction analyses showed that the positive associations of serum fT3 levels with hepatic fat were strongest among obese individuals. In that subgroup of individuals, we also observed an inverse association between serum TSH levels and hepatic fat content.

A recent meta-analysis (3), as well as longitudinal data from the Rotterdam study (4), showed a significant association between hypothyroidism and NAFLD. Likewise, previous studies also showed a positive association between serum TSH levels and NAFLD $(4,6,18)$. In contrast to the latter studies, but in line with a previous report from our study region (5), we did not find any significant association of serum TSH levels with liver fat content or NAFLD. This discrepancy may be related to the relatively low number of hypothyroid individuals in our study, which is a consequence of the long-term iodine deficiency in Germany persisting until the middle of the 1990s (19). Thus, due to the low number of individuals with high TSH levels we may have overlooked an association between serum TSH levels and NAFLD. Furthermore, our interaction analyses of TSH with BMI on hepatic fat content pointed toward a positive association between serum TSH levels and hepatic fat content in individuals with a BMI $<25$ $\mathrm{kg} / \mathrm{m}^{2}$, while in obese individuals we observed an inverse association of serum TSH levels with hepatic fat content. Thus, some discrepancies between studies might also be related to different body fat distributions of the study populations.

Previous studies diagnosed NAFLD with either ultrasonography $(5,6,18)$ or fatty liver index (4). In our study, we defined NAFLD according to MRI-PDFF,

Table 4 Associations of thyroid function with the combined endpoint of NAFLD and hepatic iron overload. Data are reported as relative risk ratios (RRR) derived from multinomial logistic regression models with base category 'no hepatic fat and no hepatic iron overload' adjusted for age, sex, alcohol consumption, BMI, physical activity, food frequency score, and time of blood sampling.

\begin{tabular}{|c|c|c|c|c|c|c|}
\hline \multirow[b]{3}{*}{ Exposure } & \multicolumn{6}{|c|}{ Combined endpoint ${ }^{a}$} \\
\hline & \multicolumn{2}{|c|}{ +Fat -iron } & \multicolumn{2}{|c|}{-Fat +iron } & \multicolumn{2}{|c|}{ +Fat +iron } \\
\hline & RRR & $P$ value & RRR & $P$ value & RRR & $P$ value \\
\hline Power-transformed TSH & $1.23(0.59,2.57)$ & 0.576 & $1.59(0.44,5.79)$ & 0.479 & $1.29(0.37,4.46)$ & 0.690 \\
\hline Power-transformed fT4 & $0.34(0.15,0.75)$ & 0.008 & $0.22(0.05,0.89)$ & 0.035 & $0.16(0.04,0.62)$ & 0.008 \\
\hline Power-transformed fT3 & $8.20(3.56,18.87)$ & $<0.001$ & $0.25(0.06,1.07)$ & 0.062 & $9.56(1.93,47.28)$ & 0.006 \\
\hline Power-transformed fT3/fT4 ratio & $13.06(5.67,30.06)$ & $<0.001$ & $1.26(0.33,4.77)$ & 0.733 & $30.51(6.96,133.7)$ & $<0.001$ \\
\hline
\end{tabular}

aCombined endpoint: NAFLD was defined by an MRI-PDFF threshold of liver fat content $\geq 5.1 \%$ plus liver iron overload was defined by a threshold $\mathrm{R}^{2 *} \geq 43.9$.

$\begin{array}{lr}\text { https://ec.bioscientifica.com } & \text { (c) } 2022 \text { The authors } \\ \text { https://doi.org/10.1530/EC-21-0566 } & \text { Published by Bioscientifica Ltd }\end{array}$

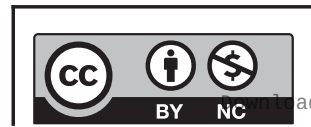

This work is licensed under a Creative Commons Attribution-NonCommercial 4.0 International License. ded from Bioscientifica com at $04 / 26 / 2023$ 10:36:52AM 

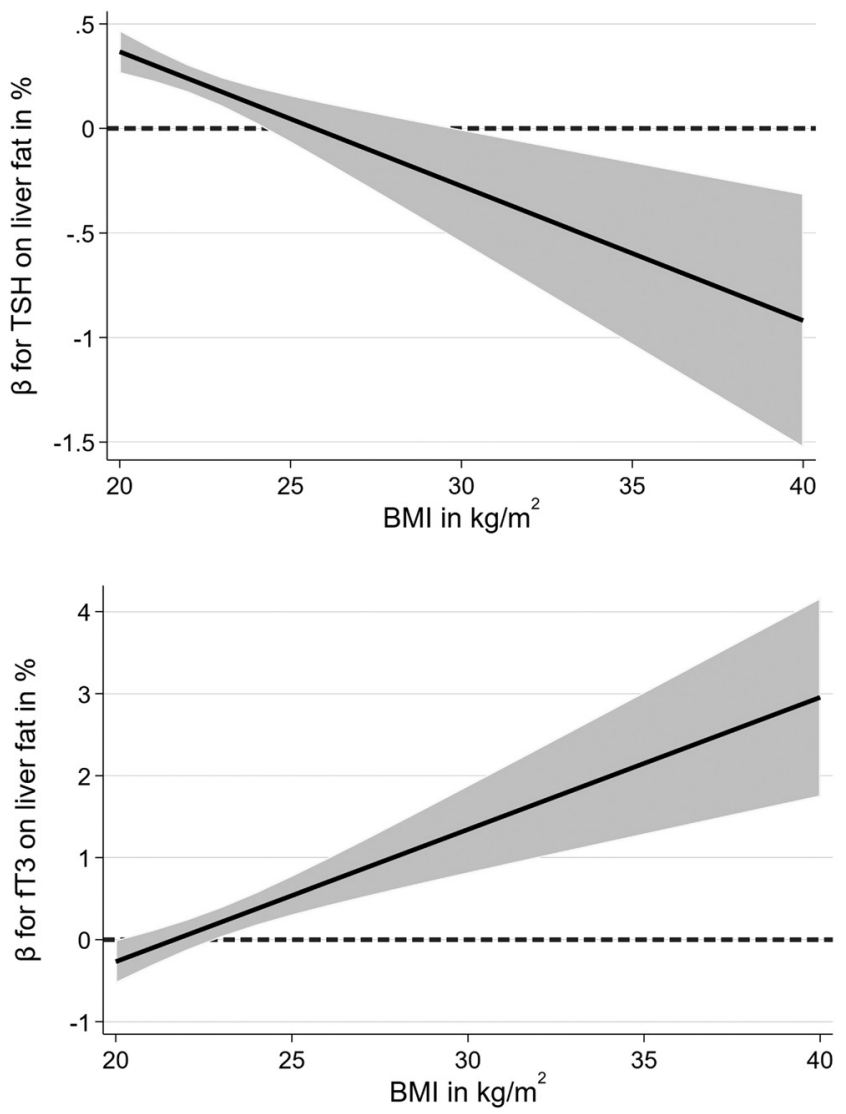

Figure 3

Associations of serum TSH and fT3 levels with hepatic fat content for different BMI values after adjustment for age, sex, alcohol consumption, physical activity, food frequency score, and time between blood sampling and MRI examinations. Results are expressed as $\beta$-coefficients and $95 \% \mathrm{Cl}$ for different BMI values.

which allows not only an accurate assessment of hepatic fat but also hepatic iron content (8). In a comparison study, we showed that hepatic steatosis, measured by ultrasound, was a very sensitive marker for hepatic steatosis determined by MRI-PDFF for individuals with moderate or severe hepatic fat content, but in the group with low hepatic fat content, the sensitivity of ultrasound was only $65 \%(20)$. Thus, the conflicting findings of our study to previous studies might to some extent be explained by different measurement techniques of hepatic fat content.

In our study, we observed inverse associations of serum fT4 levels with NAFLD and hepatic iron overload, while serum fT3 levels were positively associated with NAFLD. An inverse association between serum fT4 levels and NAFLD was also found in previous population-based studies $(4,5$, 21), of which one also showed a positive association between serum fT3 levels and NAFLD (21). These oppositional associations of fT3 and fT4 with NAFLD might be partly explained by different degrees of body fat accumulation.
A previous study involving 160 obese adolescents showed a significantly higher fT3/fT4 ratio in those with NAFLD compared to their counterparts without NAFLD (22). Likewise, serum fT3 levels were positively associated with the components of the metabolic syndrome (22), and another study showed that body weight reduction was associated with a decrease in serum fT3 levels as a putative adaptive mechanism (23). In our study population, about one-third of the individuals were obese and the observed significant associations of serum fT3 levels with liver fat content were strongest in obese individuals. Thus, our findings might be explained by a higher conversion rate of fT4 to fT3 due to increased deiodinase activity as a compensatory mechanism for increased body fat accumulation (23). Overall, the concentration of thyroid hormones may not be markedly decreased or increased, so that the pituitary gland is not affected through the negative feedback loop to alter the release of TSH. In obese individuals, however, we found an inverse association between serum TSH levels and liver fat content, which may be a consequence of the stronger association of serum fT3 levels with liver fat in obese individuals compared to individuals with normal weight or overweight.

For hepatic iron content, our results were to some extent similar to those for NAFLD, but there were no significant associations of fT3 and fT3/fT4 ratio with hepatic iron overload. Likewise, when looking at the combined endpoint of NAFLD and hepatic iron overload, we observed only for low fT 4 an increased risk for hepatic iron overload in the absence of NAFLD. Thus, our results may argue for a stronger association between thyroid hormones and NAFLD than hepatic iron overload. The underlying mechanisms explaining the associations of thyroid dysfunction with NAFLD and hepatic iron overload may be somewhat similar since thyroid hormone alterations, NAFLD, and hepatic iron accumulation are strongly related with metabolic factors, such as obesity and insulin resistance $(24,25)$.

The results of our study may stress that not only subclinical hypothyroidism but also hyperthyroidism is associated with increased hepatic fat particularly in obese individuals. However, one has to be cautious with these findings, and our results have to be further verified in longitudinal studies, which should focus on obese individuals and include participants from regions with different history of iodine supply.

One of the most important strengths of our study is its population-based design including about 2500 individuals in total. Additionally and, most importantly, we assessed liver fat and iron contents by chemical shift MRI

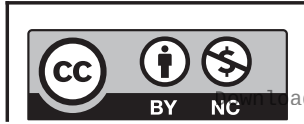


techniques for the precise quantification of NAFLD and hepatic iron overload. Liver biopsy is an invasive method and is inappropriate for screening as well as unethical for epidemiological studies $(26,27)$. Liver ultrasonography is less accurate for quantifying liver fat (especially when less of $20 \%$ of hepatocytes are steatotic) and does not allow to differentiate hepatic fat from hepatic iron (28, 29). The major limitation of our study is its cross-sectional design from which we cannot draw any causal inference. Furthermore, we had substantial dropout of individuals to MRI examinations. To account for this issue, we used inverse probability weighing.

While serum levels of fT 4 were inversely associated with NAFLD, there were positive associations of serum fT3 levels and the fT3/fT4 ratio with NAFLD. These findings might be the result of a higher conversion of fT4 to the biologically active form fT3. In obese individuals, we observed an inverse association between serum TSH levels and NAFLD, which may indicate that a subclinical hyperthyroid state is associated with NAFLD particularly in obese individuals. Furthermore, thyroid hormone levels seemed to be more strongly associated with liver fat compared to liver iron content.

\section{Declaration of interest}

The authors declare that there is no conflict of interest that could be perceived as prejudicing the impartiality of the research reported.

\section{Funding}

This work was funded in part by grants from the German Federal Ministry for Education and Research, from the Ministry for Education, Research and Cultural Affairs, the Ministry for Social Affairs of the State MecklenburgWest Pomerania.

\section{Author contribution statement}

$\mathrm{TI}$ and $\mathrm{R} \mathrm{M} \mathrm{K}$ conducted the data analyses; T I, R M K, and $\mathrm{H} V$ have written the first draft of the manuscript; TI, M R P M, J P K, M L K, A S, M N, and H V were involved in planning and conduction of the study and its assessments; all authors critically drafted and reviewed the manuscript and gave final permission for submission of the manuscript.

\section{Acknowledgements}

The Study of Health in Pomerania is part of the Community Medicine Research net of the University Medicine Greifswald, which is supported by the German Federal State of Mecklenburg-West Pomerania (http://www. community-medicine.de).

\section{References}

1 Younossi ZM, Stepanova M, Younossi Y, Golabi P, Mishra A, Rafiq N $\&$ Henry L. Epidemiology of chronic liver diseases in the USA in the past three decades. Gut 202069 564-568. (https://doi.org/10.1136/ gutinl-2019-318813)

2 Jaruvongvanich V, Sanguankeo A \& Upala S. Nonalcoholic fatty liver disease is not associated with thyroid hormone levels and hypothyroidism: a systematic review and meta-analysis. European Thyroid Journal 20176 208-215. (https://doi.org/10.1159/000454920)

3 Mantovani A, Nascimbeni F, Lonardo A, Zoppini G, Bonora E, Mantzoros CS \& Targher G. Association between primary hypothyroidism and nonalcoholic fatty liver disease: a systematic review and meta-analysis. Thyroid 201828 1270-1284. (https://doi. org/10.1089/thy.2018.0257)

4 Bano A, Chaker L, Plompen EP, Hofman A, Dehghan A, Franco OH, Janssen HL, Darwish Murad S \& Peeters RP. Thyroid function and the risk of nonalcoholic fatty liver disease: the Rotterdam study. Journal of Clinical Endocrinology and Metabolism 2016101 3204-3211. (https:// doi.org/10.1210/jc.2016-1300)

5 Ittermann T, Haring R, Wallaschofski H, Baumeister SE, Nauck M, Dorr M, Lerch MM, Meyer zu Schwabedissen HE, Rosskopf D \& Volzke H. Inverse association between serum free thyroxine levels and hepatic steatosis: results from the study of health in Pomerania Thyroid 201222 568-574. (https://doi.org/10.1089/thy.2011.0279)

6 Tahara K, Akahane T, Namisaki T, Moriya K, Kawaratani H, Kaji K, Takaya H, Sawada Y, Shimozato N, Sato S, et al. Thyroid-stimulating hormone is an independent risk factor of non-alcoholic fatty liver disease. JGH Open 20204 400-404. (https://doi.org/10.1002/igh3.12264)

7 Lugari S, Mantovani A, Nascimbeni F \& Lonardo A. Hypothyroidism and nonalcoholic fatty liver disease - a chance association? Hormone Molecular Biology and Clinical Investigation 201841 20180047. (https:// doi.org/10.1515/hmbci-2018-0047)

8 Kühn JP, Hernando D, Mensel B, Krüger PC, Ittermann T, Mayerle J, Hosten N \& Reeder SB. Quantitative chemical shift-encoded MRI is an accurate method to quantify hepatic steatosis. Journal of Magnetic Resonance Imaging 201439 1494-1501. (https://doi.org/10.1002/ jmri.24289)

9 Kühn JP, Hernando D, Muñoz del Rio A, Evert M, Kannengiesser S, Völzke H, Mensel B, Puls R, Hosten N \& Reeder SB. Effect of multipeak spectral modeling of fat for liver iron and fat quantification correlation of biopsy with MR imaging results. Radiology 2012265 133-142. (https://doi.org/10.1148/radiol.12112520)

10 Hines C, Yokoo T, Bydder M, Sirlin C \& Reeder S. Optimization of flip angle to allow tradeoffs in T1 bias and SNR performance for fat quantification. Proceedings of the International Society for Magnetic Resonance in Medicine 2010182927.

11 Macaron CI \& Macaron ZG. Increased serum ferritin levels in hyperthyroidism. Annals of Internal Medicine 198296 617-618. (https:// doi.org/10.7326/0003-4819-96-5-617)

12 Van De Vyver FL, Blockx PP, Abs RE, Van Den Bogaert WG \& Bekaert JL. Serum ferritin levels in hyperthyroidism. Annals of Internal Medicine 198297 930-931. (https://doi.org/10.7326/0003-4819-97-6-930_3)

13 Völzke H, Wallaschofski H, Wolff B, Berger K, John U \& Dörr M. Thyroid function and serum ferritin levels: the study of health in Pomerania. Thyroid 200616 681-686. (https://doi.org/10.1089/ thy.2006.16.681)

14 Khattak RM, Ittermann T, Nauck M, Below H \& Völzke H. Monitoring the prevalence of thyroid disorders in the adult population of Northeast Germany. Population Health Metrics 201614 39. (https://doi. org/10.1186/s12963-016-0111-3)

15 John U, Greiner B, Hensel E, Lüdemann J, Piek M, Sauer S, Adam C, Born G, Alte D, Greiser E, et al. Study of health in Pomerania (SHIP): a health examination survey in an east German region: objectives and design. Sozial- und Präventivmedizin 200146 186-194. (https://doi. org/10.1007/BF01324255)

16 Ittermann T, Khattak RM, Nauck M, Cordova CM \& Volzke H. Shift of the TSH reference range with improved iodine supply in Northeast Germany. European Journal of Endocrinology 2015172 261-267. (https:// doi.org/10.1530/EJE-14-0898) https://ec.bioscientifica.com https://doi.org/10.1530/EC-21-0566 (c) 2022 The authors Published by Bioscientifica Ltd
This work is licensed under a Creative Commons Attribution-NonCommercial 4.0 International License. ad from Bioscientifica com at 04/26/2023 10:36:52AM 
17 Royston P \& Sauerbrei W. Multivariable Model-Building: A Pragmatic Approach to Regression Analysis Based on Fractional Polynomials for Modelling Continuous Variables. Chichester, West Sussex: John Wiley \& Sons, Ltd, 2008. (https://doi.org/10.1002/9780470770771)

18 Chung GE, Kim D, Kwak MS, Yim JY, Ahmed A \& Kim JS. Longitudinal change in thyroid-stimulating hormone and risk of nonalcoholic fatty liver disease. Clinical Gastroenterology and Hepatology 202119848. e1-849.e1. (https://doi.org/10.1016/j.cgh.2020.02.039)

19 Volzke H, Ludemann J, Robinson DM, Spieker KW, Schwahn C, Kramer A, John U \& Meng W. The prevalence of undiagnosed thyroid disorders in a previously iodine-deficient area. Thyroid 200313 803-810. (https://doi.org/10.1089/105072503768499680)

20 Kromrey ML, Ittermann T, Berning M, Kolb C, Hoffmann RT, Lerch MM, Volzke H \& Kuhn JP. Accuracy of ultrasonography in the assessment of liver fat compared with MRI. Clinical Radiology 201974 539-546. (https://doi.org/10.1016/j.crad.2019.02.014)

21 van den Berg EH, van Tienhoven-Wind LJ, Amini M, Schreuder TC, Faber KN, Blokzijl H \& Dullaart RP. Higher free triiodothyronine is associated with non-alcoholic fatty liver disease in euthyroid subjects: the Lifelines Cohort Study. Metabolism: Clinical and Experimental 2017 67 62-71. (https://doi.org/10.1016/j.metabol.2016.11.002)

22 Bilgin H \& Pirgon Ö. Thyroid function in obese children with nonalcoholic fatty liver disease. Journal of Clinical Research in Pediatric Endocrinology 20146 152-157. (https://doi.org/10.4274/Jcrpe.1488)

23 Rosenbaum M, Hirsch J, Murphy E \& Leibel RL. Effects of changes in body weight on carbohydrate metabolism, catecholamine excretion, and thyroid function. American Journal of Clinical Nutrition $2000 \mathbf{7 1}$ 1421-1432. (https://doi.org/10.1093/ajcn/71.6.1421)

24 Malik R \& Hodgson $H$. The relationship between the thyroid gland and the liver. QJM 200295 559-569. (https://doi.org/10.1093/ qjmed/95.9.559)

25 Vernon G, Baranova A \& Younossi ZM. Systematic review: the epidemiology and natural history of non-alcoholic fatty liver disease and non-alcoholic steatohepatitis in adults. Alimentary Pharmacology and Therapeutics 201134 274-285. (https://doi.org/10.1111/j.13652036.2011.04724.x)

26 Copel L, Sosna J, Kruskal JB \& Kane RA. Ultrasound-guided percutaneous liver biopsy: indications, risks, and technique. Surgical Technology International 200311 154-160.

27 Ratziu V, Charlotte F, Heurtier A, Gombert S, Giral P, Bruckert E, Grimaldi A, Capron F, Poynard T \& LIDO Study Group. Sampling variability of liver biopsy in nonalcoholic fatty liver disease. Gastroenterology 2005128 1898-1906. (https://doi.org/10.1053/j. gastro.2005.03.084)

28 Dasarathy S, Dasarathy J, Khiyami A, Joseph R, Lopez R \& McCullough AJ. Validity of real time ultrasound in the diagnosis of hepatic steatosis: a prospective study. Journal of Hepatology 200951 1061-1067. (https://doi.org/10.1016/j.jhep.2009.09.001)

29 Strauss S, Gavish E, Gottlieb P \& Katsnelson L. Interobserver and intraobserver variability in the sonographic assessment of fatty liver. American Journal of Roentgenology 2007189 W320-W323. (https://doi. org/10.2214/AJR.07.2123)

Received in final form 13 January 2022

Accepted 21 January 2022

Accepted Manuscript published online 21 January 2022
This work is licensed under a Creative Commons Attribution-NonCommercial 4.0 International License. ded from Bioscientifica.com at 04/26/2023 10:36:52AM 